\title{
Au(111): A theoretical study of the surface reconstruction and the surface electronic structure
}

\author{
Noboru Takeuchi, C. T. Chan, and K. M. Ho \\ Ames Laboratory and Department of Physics, Iowa State University, Ames, Iowa 50011
}

(Received 6 December 1990)

\begin{abstract}
On the reconstructed $\mathrm{Au}(111)$ surface, atoms on the surface layer occupy both the hcp and the fcc sites. Using first-principles calculations to obtain the surface energies of the system with the top Au layer occupying the fcc, hcp, top, and bridge sites, we found that the hcp site is only $1 \mathrm{mRy}$ per surface atom higher in energy than the fcc site. The complex Au(111) reconstruction is then discussed with use of a two-dimensional Frenkel-Kontorowa model. We calculated the surface band structure of the $A u(111)$ surface along high-symmetry lines in the surface Brillouin zone, with the top layer occupying the fcc, hcp, and bridge sites. We found that the surface electronic structure is almost independent of the position of the top layer.
\end{abstract}

\section{INTRODUCTION}

Clean metal surfaces may have a structure that is not a simple termination of the bulk. This phenomenon, called reconstruction, is seen in all the low-index surfaces of Au. ${ }^{1}$ The $(100)$ surface exhibits a $c(26 \times 68)$ pattern, the (110) surface shows a $(1 \times 2)$ reconstruction, and the (111) surface reconstructs with a $[(22 \pm 1) \times \sqrt{3}]$ surface unit cell. The case of the (111) surface is particularly interesting because gold is the only clean face-centered-cubic (fcc) metal with a (111) surface that has a reconstructed ground state. This reconstruction was well-studied by many techniques, including low-energy electron diffraction (LEED), 1,2 reflection high-energy electron diffraction (RHEED), ${ }^{3}$ and transmission electron diffraction (TED). ${ }^{1,5}$ Van Hove et al. ${ }^{1}$ proposed a model of a $(p \times \sqrt{3})$ structure with $p=21-22$, with a surface layer contraction in one of the three equivalent [1 10 ] directions. High-resolution transmission electron diffraction experiments conducted by Takayanagi and Yagi $^{5}$ suggested that the compression is not uniform, but is localized in two transition regions where the stacking changes abruptly from $A B C$ to $A B A$. Harten, Lahee, and Toennies studied this reconstruction with heliumatom diffractions. ${ }^{6}$ Their experiment gave more information about the $A B C$ and $A B A$ stacking, and the boundary regions between the two of them. They interpreted the diffraction pattern as a manifestation of a onedimensional sine-Gordon solitonlike misfit structure. However, in the same experiment, they showed that the width of the regions with $A B A$ and $A B C$ stacking are not equal. A single sine-Gordon model assumes equally spaced solitons, and so cannot describe two regions with different widths. El-Batanouny et $a l .{ }^{7}$ proposed a double sine-Gordon model that allows for the existence of $A B C$ and $A B A$ stacking with different domain sizes.

In most of these models both the hexagonal-closepacked (hcp) sites and the face-centered-cubic sites are occupied by the surface atoms. Experimentally, this has been shown in scanning tunneling microscopy (STM) experiments. ${ }^{8}$ A necessary condition for this to happen is that the fcc and hcp sites have very similar energies for top-layer $\mathrm{Au}$ atoms. Using first-principles total-energy calculations, we show that the two sites differ in energy by only $1 \mathrm{mRy}$ per surface atom. Another necessary condition for the $\mathrm{Au}(111)$ reconstruction is the tendency of the top layer to contract to a higher density than in an ideal (111) surface layer. Our previous calculations have demonstrated that this is possible. ${ }^{9}$ Using the information that we have about the top-layer contraction, ${ }^{9}$ and the energy of occupancy of various surface sites computed by total-energy techniques, the surface reconstruction is described with a two-dimensional Frenkel-Kontorowa (FK) model. ${ }^{10}$

The STM measurements of Wöll et al. ${ }^{8}$ confirmed previous models of the reconstruction, and at the same time provided images of the real-space atomic arrangement of the surface with atomic resolution. The fact that the STM is giving images with atomic resolution for the surfaces of a metal is slightly surprising and may suggest the existence of surface electron state near the Fermi level that give larger corrugation than the overall charge density. We investigated the surface electronic structure of the (111) surface of Au along the $\Gamma-M-K-\Gamma$ lines, together with the projected bulk band structure on the twodimensional Brillouin zone. The rest of the paper is organized as follows: the first-principles techniques used in our calculations are described in Sec. II, the calculation of the energies for the different sites are in Sec. III, the simulation with the two-dimensional Frenkel-Kontorowa model is described in Sec. IV, the surface bands and the projected band structures are presented in Sec. V, and the summary of the paper is in Sec. VI.

\section{FIRST-PRINCIPLES CALCULATIONS}

We use in our calculations nonlocal ionic pseudopotentials generated using the norm-conserving scheme of Hamann, Schlüter, and Chiang. ${ }^{11}$ The total energies are calculated within the local-density-functional formalism ${ }^{12}$ with the Hedin-Lundqvist ${ }^{13}$ form of the local exchangecorrelation energy and potential. The wave functions are 
expanded by means of an efficient mixed basis set consisting of planes waves with kinetic energy $(\mathbf{k}+\mathbf{G})^{2}$ up to certain $E_{\text {cut }}$ and a set of localized functions centered at the atomic sites to describe the more tightly bound $d$ orbitals. ${ }^{14}$ For the calculation of the energies of the different sites, we use $E_{\text {cut }}=12 \mathrm{Ry}$ and a set of Gaussian as local orbitals. For a given $E_{\text {cut }}$, the decay coefficient of the local Gaussian orbital is determined variationally from bulk calculations to optimize the convergence of the basis set. A slab consisting of seven Au (111) layers is used to describe the surface. For the surface-state calculations, the number of $\mathrm{Au}$ layers is increased to 15 to facilitate the identification of surface states. We found that, using numerical functions to represent the local orbitals, a cutoff of $7.5 \mathrm{Ry}$ for the plane-wave expansion is sufficient to provide accurate surface band-structure results. ${ }^{15}$ The pseudopotential mixed-basis approach has been used in previous investigations of the structural properties of bulk $\mathrm{Au},{ }^{16}$ and the reconstruction of the $\mathrm{Au}(110)$ (Ref. 17) and $\mathrm{Au}(100)$ (Ref. 9) surfaces, with excellent results.

\section{III. $A B C$ AND $A B A$ PACKING}

The face-centered-cubic and the hexagonal-closepacked structures represent two different ways of arranging hard spheres in a regular array to minimize the interstitial volume. The fcc structure corresponds to an $A B C \cdots$ packing of hexagonal layers of atoms, while the hcp structure corresponds to an $A B A \cdots$ packing. For the $\mathrm{Au}(111)$ surface, if a continuation of the bulk structure occurs, an $A B C$ stacking is expected ( $\mathrm{Au}$ is an fcc metal). However, the $\mathrm{Au}(111)$ surface reconstructs to a $(22 \pm 1) \times \sqrt{3}$ superlattice. Previous studies of the energetics of a Au monolayer on top of a uniform substrate ${ }^{9}$ show that a single layer of $\mathrm{Au}$ atoms favors contraction to a smaller lattice constant from the bulk value. The intrinsic tendency for the Au surface layer to contract to higher surface density is opposed by the substrate potential, which tries to keep the top layer in registry ( $A B C$ stacking) with the underlying atomic layers. However, if the energy required to occupy the hcp sites ( $A B A$ stacking) is close to the energy required to occupy the fcc sites, the energy cost in losing registry is expected to be small and a contraction of the top layer can be favorable.

We use first-principles techniques to obtain the total energy of the top Au layer occupying the fcc sites and the hcp sites (labeled $C$ and $A$, respectively, in Fig. 1). The energies for the top site $T$ and the bridge site $B$ are also obtained. For the fcc, hcp, and top-site calculations, an evenly spaced sampling grid of $15 \mathbf{k}$ points in the irreducible part of the surface Brillouin zone (SBZ) [Fig. 2(a)] is used for the calculation of the electronic charge density during the iteration to self-consistency. In the case of the bridge site, the sampling grid is increased to $45 \mathrm{k}$ points due to reduced symmetry [see Fig. 2(b)]. All the interlayer distances are fully relaxed using forces calculated by the Hellmann-Feynman theorem. For the unreconstructed $\mathrm{Au}(111)$ surface, we found the surface energy to be $1.04 \mathrm{~J} / \mathrm{m}^{2}$. The fcc sites have the lowest energy. Results

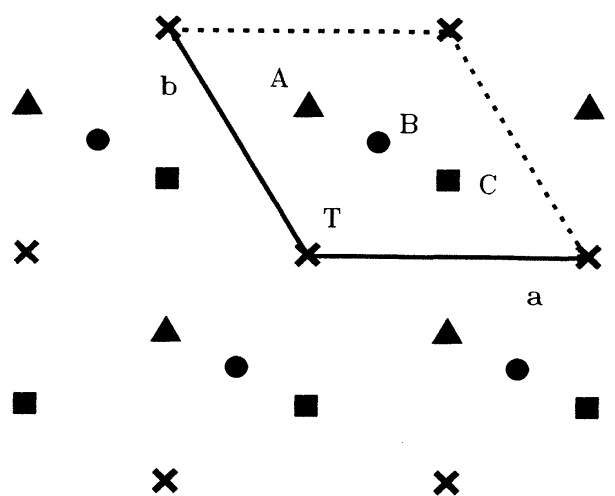

FIG. 1. A schematic top view of the (111) face of Au. The symbols $A, C, T$, and $B$ label atoms in the hcp, fcc, top, and bridge sites, respectively.

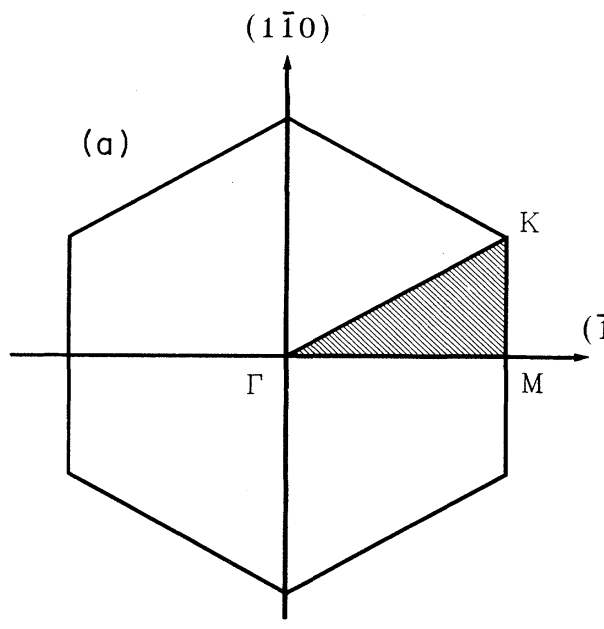

(1̄i2)

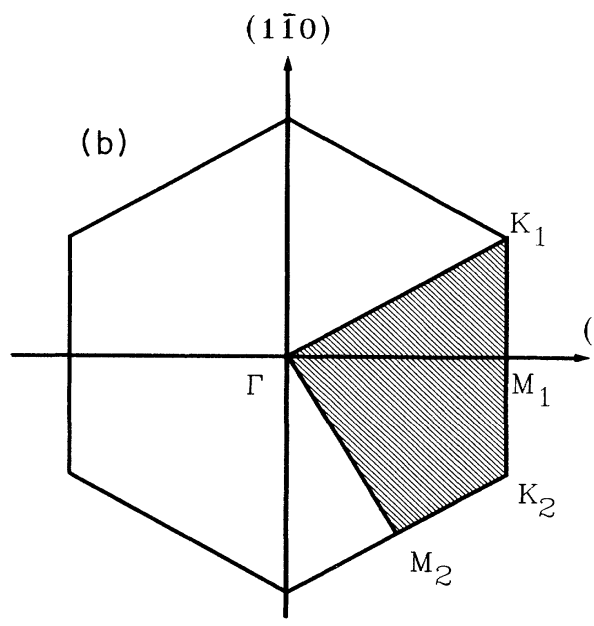

$(\overline{1} \overline{1} 2)$

FIG. 2. (a) The 2D Brillouin zone of $\mathrm{Au}(111)$ for the surface atom at the fcc, hcp, and top sites; (b) the 2D Brillouin zone of $\mathrm{Au}(111)$ for the surface atom in the bridge site. The irreducible parts of the Brillouin zones are shaded. 
TABLE I. Energy per surface atom for the top layer occupying different sites, using the fcc site as reference.

\begin{tabular}{lc}
\hline Site & Energy $(\mathrm{mRy})$ \\
\hline fcc & 0 \\
hcp & 1 \\
Top & 14 \\
Bridge & 3 \\
\hline \hline
\end{tabular}

comparing the energies of the various sites are summarized in Table I. The surface energies quoted are in units of $\mathrm{mRy}$ per surface atom, and the zero of the energy is taken to be the energy of the top layer occupying the fcc site. We observe that indeed the energy of the hcp site is very close to the energy of the fcc site. The small energy difference is consistent with the observed occupation of both sites. We can also see that the energy difference between the fcc site and the bridge site is only about $3 \mathrm{mRy}$. This is a small number compared with the energy difference with the top site (14 mRy for the fcc site and $13 \mathrm{mRy}$ for the hcp site). This fact allows a gradual shift of the atoms from the fcc to the hcp stacking through the bridge site $B$, and a contraction of the surface layer along

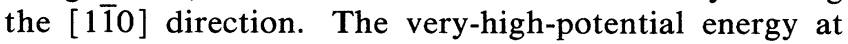
the top site makes the contraction of the lattice in the $[11 \overline{2}]$ direction very unfavorable in energy. Hence, it is rather natural that the reconstruction observed is uniaxial rather than a uniform contraction.

In Table II, we show the relaxation of the interlayer separations in the direction normal to the (111) surface (in percentages of the ideal bulk separation) for the top Au layer occupying different positions. We observe that in all cases, only the first layer shows a significant relaxation. From the vertical position of the surface atoms at the hollow sites ( $A$ or $C$ ) and the one at the bridge site, we can estimate a corrugation height of the order of 0.05 $\AA$ on the reconstructed surface. This is smaller than the $0.15 \AA$ found experimentally. ${ }^{8}$ However, this should not be regarded as a large discrepancy. The corrugation, whether deduced by theory or experiment, is rather small anyway, and closer examination of published experimental data (e.g., Fig. 2 of Ref. 8) would reveal that the 0.15 $\AA$ quoted is more like an order-of-magnitude estimate.

In the above calculation, the surface is modeled by periodic slabs seven layers thick separated by an empty space of a thickness equivalent to four layers. The adequacy of the vacuum spacing between the slabs has been tested. We increase the vacuum distance to six layers and repeat the calculations with the top layer of $\mathrm{Au}$ atoms in the fcc and bridge sites, respectively. The difference in the energy per surface atom between the two sites remains the same $(3 \mathrm{mRy})$. We have also tested the thickness of the slab. Increasing the number of Au layers to nine does not give any significant change. The interlayer distances with the top layer at the fcc and the bridge sites computed with different slab thickness and separation are compared in Table III. Considering the
TABLE II. Change of interlayer separations in percentage of the bulk interlayer separation for the top layer at various sites.

\begin{tabular}{lrrr}
\hline & Top & Bridge & fcc or hcp \\
\hline$\Delta_{12}$ & 16.2 & 3.7 & 1.5 \\
$\Delta_{23}$ & -0.1 & -0.3 & -0.3 \\
\hline \hline
\end{tabular}

dependence of our results on the slab thickness, vacuum thickness, and basis convergence, we found that the calculated interlayer separations are reliable to about $1 \%$.

\section{FRENKEL-KONTOROWA MODEL}

While the first-principles results shown in Sec. III offer valuable insight and information about the energetics of the $\mathrm{Au}(111)$ reconstruction, it is difficult to give a complete description of the reconstruction based on $a b$ initio calculations alone because of the large size of the reconstructed surface unit cell. However, a good description of the reconstruction can be obtained using a simple two-dimensional Frenkel-Kontorowa model, with parameters extracted from our first-principles results. The two-dimensional FK model describes a situation with two competing interactions: the top-layer interactions favor a uniform contraction to a smaller lattice constant, while the underlying potential due to the substrate atoms tries to pin the surface atoms in registry. The surface will transform if the energy gained by contraction is larger than the energy lost in losing registry with the substrate atoms, and if the surface does transform, the final atomic arrangement will be governed by the interplay between the strain energy in the top layer and the potential energy due to the underlying layers.

Our previous calculations ${ }^{9}$ show that a $\mathrm{Au}$ monolayer prefers a higher packing density than the arrangement of the surface atoms on a $\mathrm{Au}(111)$ surface with bulk lattice constant. It remains to be tested whether the tendency for contraction is large enough to overcome the potential due to the substrate.

Since the effect of the substrate atoms can be expressed in the form of a periodic potential with the symmetry and periodicity of the unreconstructed substrate surface (with the bulk lattice parameter), we expand the potential energy per surface atom in a Fourier series of the form

$$
E(\mathbf{R})=\sum_{\mathbf{G}} W_{\mathrm{G}} e^{i \mathrm{G} \cdot \mathbf{R}}
$$

where the G's are the two-dimensional (2D) reciprocallattice vectors of the substrate surface and $R=(x, y)$ is the position of an atom on the top layer.

Our knowledge of the binding energies at a few highsymmetry sites (top, bridge, fcc, and hcp) allows us to determine the first few terms in Eq. (1). Explicitly, we have 
TABLE III. Percentage relaxation of the interlayer separations for different sizes and separations of the slab.

\begin{tabular}{ccccccc}
\hline \hline & \multicolumn{2}{c}{$\begin{array}{c}\text { Seven layers of Au } \\
\text { Four layers of vacuum } \\
\text { fcc }\end{array}$} & Bridge & \multicolumn{2}{c}{$\begin{array}{c}\text { Seven layers of Au } \\
\text { Six layers of vacuum }\end{array}$} & \multicolumn{2}{c}{$\begin{array}{c}\text { Nine layers of Au } \\
\text { Four layers of vacuum } \\
\text { fcc }\end{array}$} & $\begin{array}{c}\text { Bridge } \\
\text { fcc }\end{array}$ & Bridge \\
\hline$\Delta_{12}$ & 1.5 & 3.7 & 0.8 & 3.7 & 1.6 & 2.1 \\
$\Delta_{23}$ & -0.3 & -0.3 & -0.3 & -0.3 & 0.2 & 0.3 \\
$\Delta_{34}$ & 0 & 0 & 0 & 0 & 0.1 & -0.1 \\
$\Delta_{45}$ & & & & & 0 & 0 \\
\hline
\end{tabular}

$$
\begin{aligned}
E(x, y)= & \frac{3 V_{B}}{4}-\frac{\left(V_{C}+V_{A}\right)}{9}\left[\cos \left[\frac{2 \pi}{a} x\right]+\cos \left[\frac{2 \pi}{a} y\right)+\cos \left[\frac{2 \pi}{a}(x+y)\right]\right] \\
& +\frac{\left(V_{C}-V_{A}\right)}{3 \sqrt{3}}\left[\sin \left[\frac{2 \pi}{a} x\right]+\sin \left[\frac{2 \pi}{a} y\right]-\sin \left(\frac{2 \pi}{a}(x+y)\right]\right] \\
& +\left[\frac{V_{C}+V_{A}}{9}-\frac{V_{B}}{4}\right]\left[\cos \left(\frac{2 \pi}{a}(2 x+y)\right]+\cos \left(\frac{2 \pi}{a}(2 y+x)\right]+\cos \left(\frac{2 \pi}{a}(x-y)\right]\right],
\end{aligned}
$$

where $V_{C}$ is the energy at the $C$ site, $V_{A}$ is the energy at the $A$ site, $V_{B}$ is the energy at the bridge site, and the zero of the potential energy is taken to be the energy at the top site. The lattice constant of the $2 \mathrm{D}$ hexagonal lattice is given by $a$. The Hamiltonian of the corresponding 2D FK model is

$$
H=\sum_{i, j} e\left(\mathbf{R}_{i}-\mathbf{R}_{j}\right)+\sum_{i} E\left(x_{i}, y_{i}\right),
$$

where $\mathbf{R}_{i}=\left(x_{i}, y_{i}\right)$ is the position of the $i$ th surface atom. The first term describes the interaction of the top $\mathrm{Au}$ atoms under the influence of the substrate, and this is the term that favors the contraction of the surface. Since the relative energy differences in occupying different positions with respect to the underlying layers are taken care of by the second term in Eq. (3), the first term can be deduced from the energetics of a monolayer on top of a smooth surface that mimics the average effect of the substrate. We obtain this interlayer interaction from our previous calculations of the energetics of a Au monolayer on top of a uniform background. ${ }^{9}$ In these calculations we put a layer of $\mathrm{Au}$ atoms on a jellium surface, with the jellium density determined by the electron density of $\mathrm{Au}$ midway between two nearest neighbors. The distance of the monolayer from the jellium edge was determined by energy minimization, and the energy of the jellium slab was subtracted so our final energy describes the atomic interactions within the surface layer in the background of a decaying electron sea. The function $e\left(\mathbf{R}_{i}-\mathbf{R}_{j}\right)$ is plotted in Fig. 3(a), where we also mark the interatomic distance on an unreconstructed surface. It is clear from the figure that as far as the intralayer interaction is concerned (under the average effect of the substrate), the top layer would prefer to contract to a higher surface density arrangement.

The second term in Eq. (3) is the potential imposed on the top layer of the $\mathrm{Au}$ atoms by the underlying layers, and it is described in Eq. (2), with all the parameters $\left(V_{A}, V_{B}\right.$, etc.) coming from our first-principles calculations. We note that $E(x, y)$, as formulated in Eq. (2), does not describe the total interaction potential energy of the top Au layer with the substrate, but rather the relative energy change as the atoms in the top layer change from one site to another. In Fig. 3(b), this relative potential energy per surface atom, $E(x, y)$, is plotted as a function of the position. The $x$ axis and the $y$ axis are chosen to be the [ $1 \overline{1} 0]$ and $[11 \overline{2}]$ directions respectively. Figure 3(c) shows a corresponding contour plot of the same function in the (111) plane. From these plots, the big difference in energy between the top site and the other sites is conspicuous and we can also see rather clearly low-energy "channels" spanning the fcc, bridge, and hcp sites along which the top layer can contract with minimal potential-energy loss. The ground-state configuration of the system is determined using numerical techniques. In our calculations a mesh of $119 \times 119$ atoms is used. The positions of the atoms, $\mathbf{R}_{i}$ 's are relaxed by an iterative procedure until a configuration that gives a local minimum is reached. Initially all the atoms are placed in the fcc site [i.e., in an ideal (111) surface configuration]. We find the forces that act on each atom, and then displace them in the direction of the force by an amount proportional to the force (the proportional constant is chosen variationally). We repeat this process until the force is zero. The atomic arrangement of the ground state of our FK simulation is shown in Fig. (4), where the surface $\mathrm{Au}$ atoms are represented by filled circles, and the unreconstructed second-layer atoms are shown as crosses, for reference. Our results indicate that it is indeed energetically favorable for the surface atoms on $\mathrm{Au}(111)$ to contract to a higher packing density. The reconstructed surface pattern, as shown in Fig. 4, agree nicely with the 

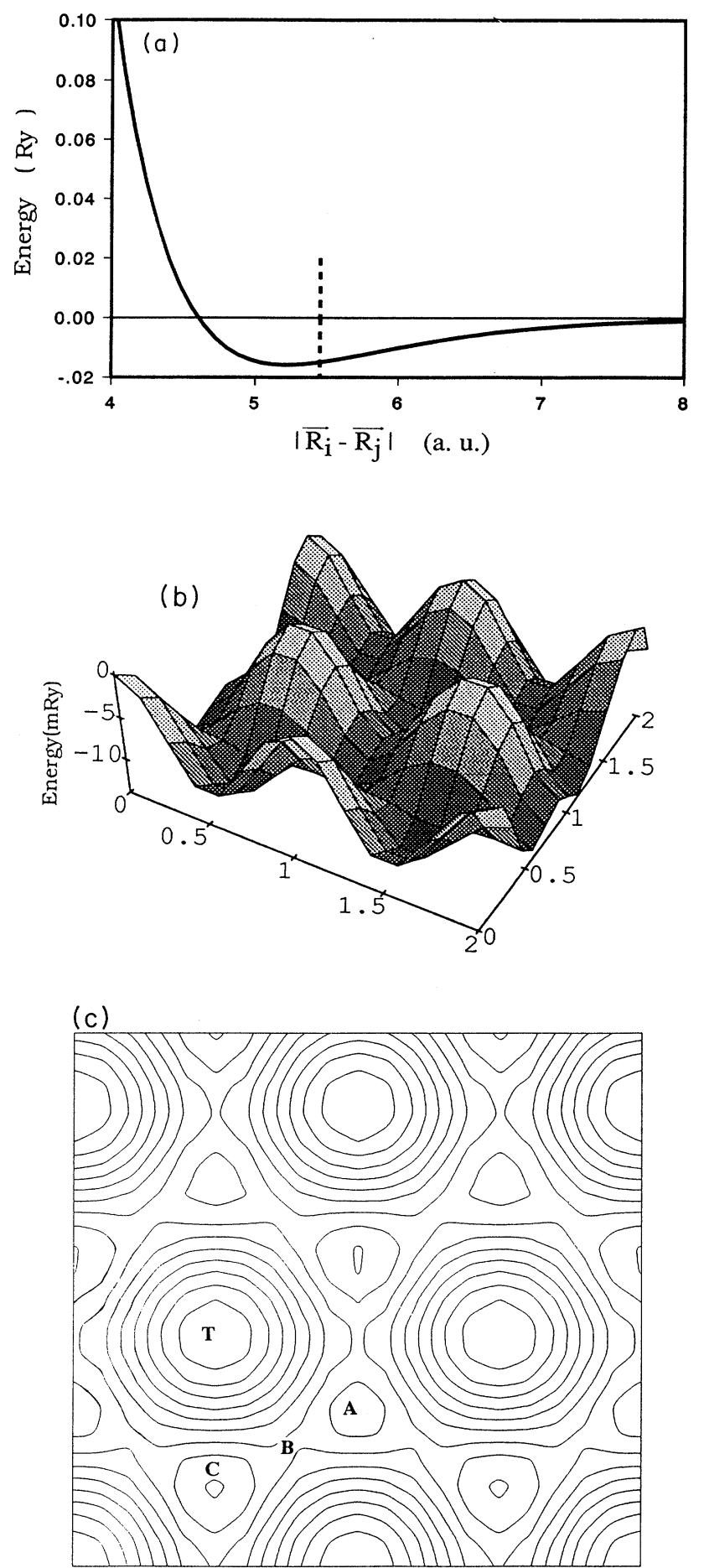

FIG. 3. (a) The intralayer interaction potential $e\left(\mathbf{R}_{i}-\mathbf{R}_{j}\right)$ [the first term in Eq. (3)]. The dotted line marks the interatomic distance on an ideal unreconstructed (111) surface. (b) A threedimensional plot of the potential energy per surface atom experienced by the top layer as a function of position [the second term in Eq. (3)]. (c) Contour plot of the potential energy per surface atom in the (111) plane. $A, C, T$, and $B$ label atoms in the hcp, fcc, top, and bridge sites, respectively. The contour interval is $1-5 \mathrm{mRy}$.

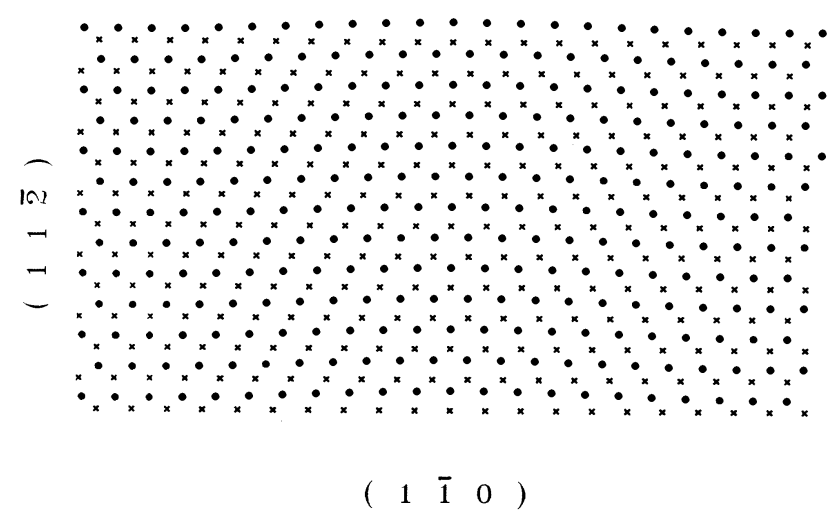

FIG. 4. The reconstructed $\mathrm{Au}(111)$ surface as given by the FK model. The crosses denote the positions of the unreconstructed second-layer atoms and the filled circles denote the positions of atoms in the reconstructed top layer.

patterns deduced from experiments. This shows that the 2D FK model, though simple, contains the essence of the physics underlying this particular problem. Furthermore, all the parameters needed are determined by theoretical computations. A closer examination of Fig. 4 shows that the surface layer does not contract to form a uniformly contracted hexagonal layer, but rather, the surface atoms contract along the [10] direction. In fact, we may view an ideal (111) surface as composed of linear rows of atoms in the [1 $1 \overline{1} 0]$ direction, and the reconstruction causes each [110] row of atoms to distort into a sinusoidal-like pattern, lying above and sandwiched by two rows of atoms in the second layer. The lattice constant in the [1 $1 \overline{1} 0]$ direction is reduced by $5.0 \%$, close to the experimentally observed $4.3 \%$ contraction. With this contraction our unit cell is a $(20 \times \sqrt{3})$ superlattice.

Both the fcc and the hcp sites are occupied and the reconstructed pattern reflects a gradual shift from one site to another. This is a consequence of the fact that the fcc and hcp sites have almost the same energy of occupation and the bridge site (midway between fcc and hcp sites) is only slightly higher in energy (see Table I and Fig. 3) so that the system finds it easier to contract along a [110] "channel," occupying the fcc, hcp, and all intermediate sites. Note that the surface does not contract in the $[11 \overline{2}]$ direction, since some surface atoms would have to climb above the top site, which is very unfavorable in energy (Table I and Fig. 3). Recent experiments ${ }^{18,19}$ have indicated that the system relaxes the surface stress in the $[11 \overline{2}]$ direction by forming ordered patterns of different domains over long distances: There are three equivalent [110] directions on the surface, so that three types of domains of equal energies can be formed on the surface, separated by domain boundaries. Recent STM (Ref. 18) and $\mathrm{x}$-ray-scattering experiments ${ }^{19}$ indicated that at room temperature these domain boundaries are ordered parallel to each other with a separation of $\approx 150 \AA$. In our simulations, only a single domain was found, since our system is relatively small in size. The $119 \times 119$ mesh is 


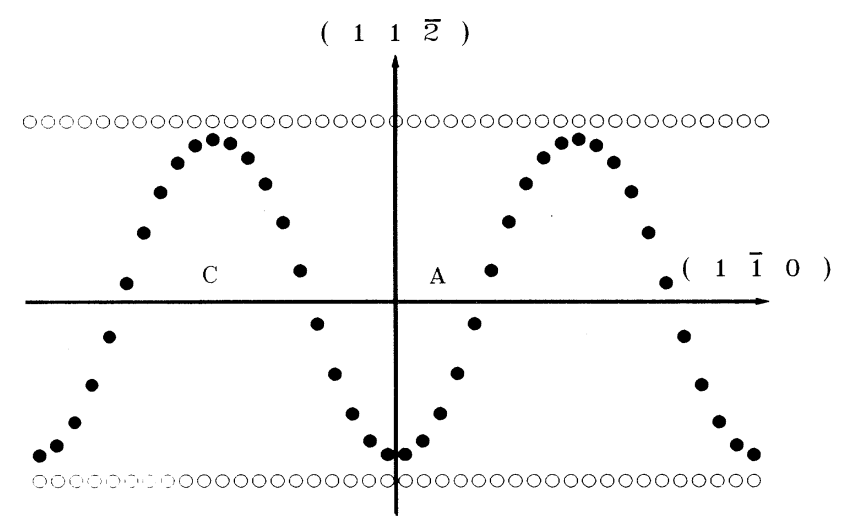

FIG. 5. One row of surface atoms on the reconstructed

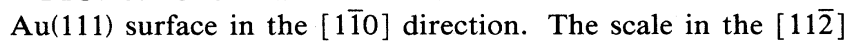
direction has been exaggerated. Filled circles denote the position of the reconstructed top layer, open circles at the top denote the positions of fcc sites, and open circles at the bottom denote the position of the hcp sites. $C$ and $A$ mark the regions of $A B C$ and $A B A$ stacking, respectively.

large enough to simulate the reconstruction in the local scale, but larger sample sizes will be needed to observe domain structures, and algorithms like simulated annealing should be preferred over the steepest decent method used in the present calculation.

Data from He scattering and STM experiments indicated that there are more surface atoms in fcc $(A B C)$ stacking than in hep ( $A B A$ ) stacking. This is consistent with the fact that the calculated energy is lower for the fcc than the hcp sites. However, different experimental techniques did not seem to agree on the exact ratio between the size of the regions with fcc and hcp stacking. This discrepancy can be partly attributed to the different assumptions used in the analysis of the experimental data. Even for a direct real-space imaging technique like STM, the image is a convolution of topological and electronic information.

It is interesting to display the reconstruction pattern from our simulation in greater detail. In Fig. 5 we plot one row of atoms (filled circles), exaggerating the scale in the $[11 \overline{2}]$ direction. The empty circles at the top represent the fcc sites in the ideal surface, and the empty circles at the bottom represent the position of the hcp sites. We observe from Fig. 5 that the pattern is more sinusoidal-like than square-wave-like, so that it is difficult to define the "size" of regions corresponding to fcc or hcp stacking for the pattern we have obtained. If we define, for the purpose of discussion, that sites that lie closer to the ideal fcc sites than the hcp sites belong to the region of fcc stacking, then there are indeed more atoms in the fcc than in the hcp stacking.

\section{SURFACE STATES}

The fact that the scanning tunneling microscope gives atomic resolution images for $\mathrm{Au}(111)$ is interesting since $\mathrm{Au}$ is a metal and the (111) surface is a compact surface, so that the charge-density profile should be very smooth at a few angstroms above the surface. This suggests that there may exist surface states near the Fermi energy, with interesting nodal structures that give rise to the atomic resolution in STM experiments. It is also interesting to investigate the effect of the surface stacking sequence on the surface electronic structures. In particular, we want to see the influence of the position of the layer on the existence, the energy, and the dispersion of the surface states. We are thus motivated to calculate the surface band structure of the $\mathrm{Au}(111)$ surface, not only for the ideal situation with the top layer in the fcc stacking sequence, but also for the top-layer atoms occupying the hcp, and the bridge sites, respectively. For better and easier identification of the surface states or resonances, we increased the slab thickness from seven layers (which has been fully relaxed using Hellmann-Feynman forces) to 15 layers by inserting eight extra layers (with bulk interlayer distances) in the middle of the slab. A vacuum equivalent to five atomic layers separates the periodic slabs. The self-consistent potentials are obtained by iterating with $15 \mathrm{k}$ points in the irreducible $\mathrm{SBZ}$ for the $\mathrm{Au}$ top layer at the fcc, and hcp, sites $(45 \mathrm{k}$ points are used for the top layer at the bridge sites, which has less symmetry). After reaching self-consistency, the electronic band structures of the systems are calculated along the symmetry directions $\Gamma-M-K-\Gamma$ for the fcc and hcp sites and along $\Gamma-M_{1}-K_{1}-\Gamma-M_{2}-K_{2}-\Gamma$ for the bridge site. ${ }^{20}$ To facilitate the identification of the surface states, we also calculate the projected band structure for $\mathrm{Au}(111)$ surface. This is done by projecting the bulk band structure of $\mathrm{Au}$ onto the two-dimensional (111) SBZ. Results are shown in Figs. 6-8, where the zero of the energy corresponds to the Fermi energy. The shaded areas indicate regions where bulk states can exist. For the top layer at the fcc and hep sites, the Hamiltonian matrix has reflection symmetry about the plane perpendicular to the (111) surface that passes along the $\Gamma-M$ line. For the case of top-layer atoms at the bridge site, this symmetry occurs only in the plane that passes along the $\Gamma-M_{2}$ line

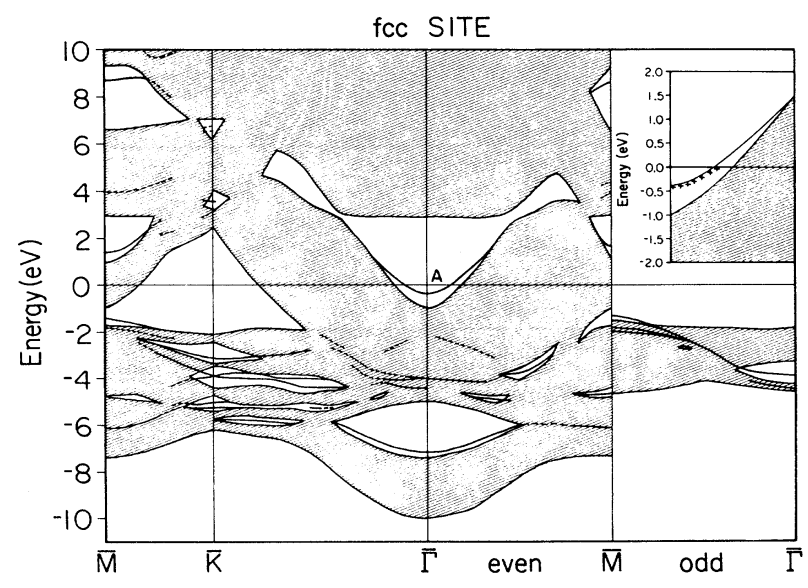

FIG. 6. Surface states and resonances along high-symmetry lines for $\mathrm{Au}(111)$ with the surface-layer atoms at the fcc sites. The inset compares the dispersion of the surface state labeled $A$ with photoemission data marked by crosses. 


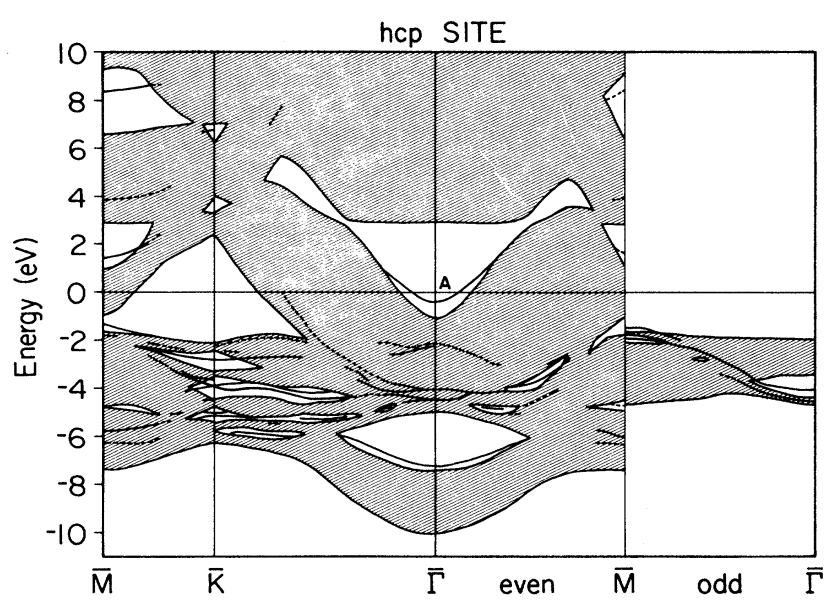

FIG. 7. Surface states and resonances along high-symmetry lines with the surface atoms at the hcp sites.
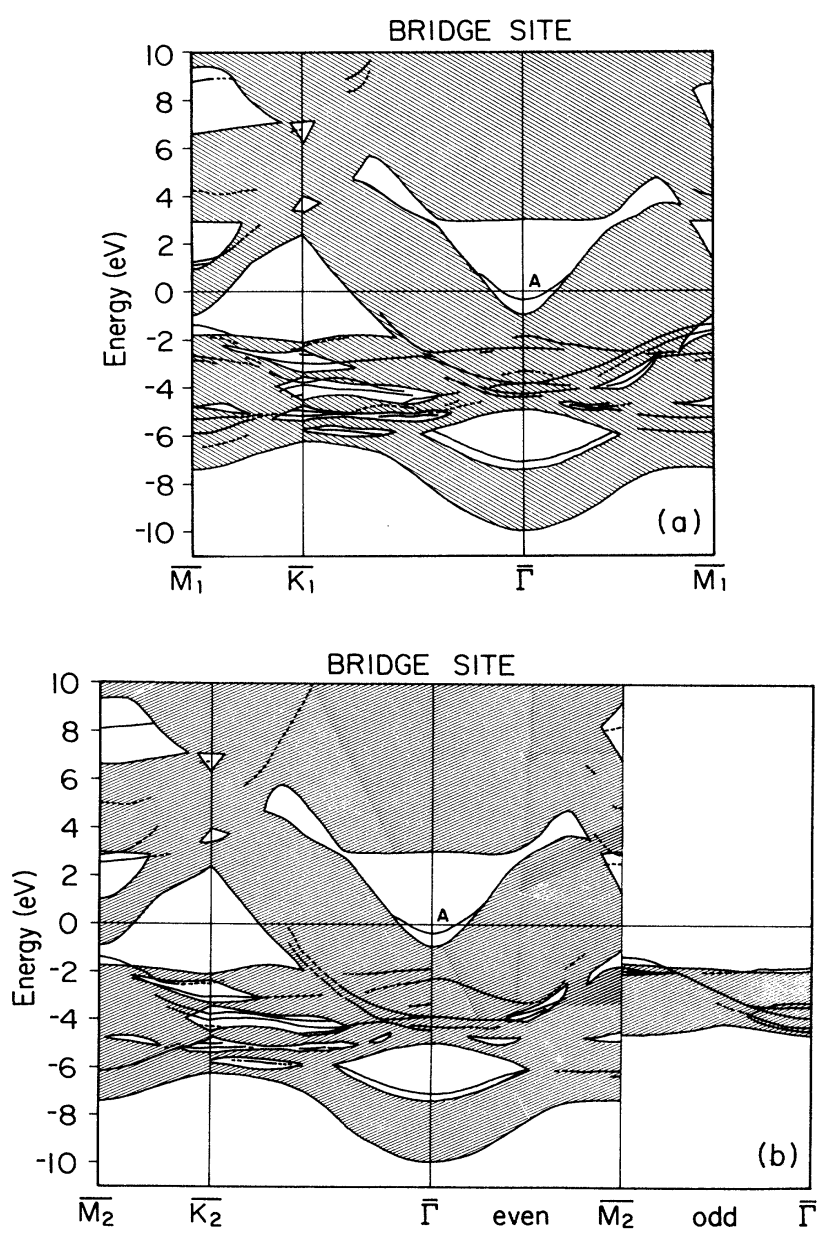

FIG. 8. Surface states and resonances along high-symmetry lines with the surface atoms at the bridge site (a) along the $M_{1}-K_{1}-\Gamma-M_{1}$ and (b) along the $M_{2}-K_{2}-\Gamma-M_{2}$. but not in the $\Gamma-M_{1}$ line. Whenever reflection symmetry exists, the electronic states with wave vectors along these symmetry lines can be separated into two groups with even and odd symmetries, and they are plotted separately in Figs. 6-8.

To locate the surface states and resonances in the surface band structure, we look for states with wavefunction amplitudes highly localized (more than 50\%) within the first two surface layers. Such states are displayed in Figs. 6-8 as solid or dashed lines according to the degree of localization at the surface. By comparing Figs. 6-8, we observe that the surface band structures along the high-symmetry lines are very similar for the top layer occupying fcc, hcp, and bridge sites. Most of the surface states and resonances that exist on the surface with the top layer in fcc stacking have corresponding surface states and resonances that exist on the surface with the top layer at the hcp and bridge sites. The dispersion of the surface states is also very similar. A closer examination reveals that even the charge densities of the corresponding surface states are very much alike. As an example, we show here a surface state at $\Gamma$ (the state marked as $A$ in the plots) near the Fermi level. This surface state has been observed by photoemission experiments ${ }^{21}$ and is probably the one probed by STM experiments. The fact that the energy and dispersion of the surface state do not depend very much on whether the top layer is in the fcc, hcp, or bridge site allows us to compare with the experimental results. This is shown in the inset in Fig. 5. We observe that our calculations agree very well with the experimental results. In Fig. 9, we plot the charge-density contours of this surface state at the $\Gamma$ point in the plane

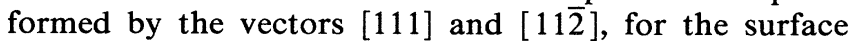
atoms occupying the fcc, hcp, and bridge sites. The posi-

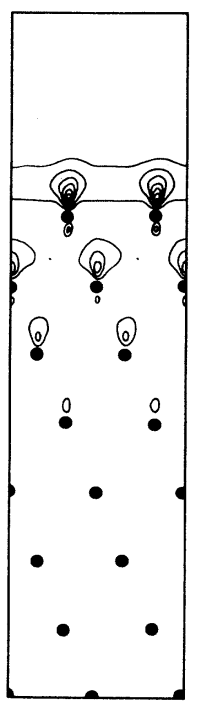

fcc site

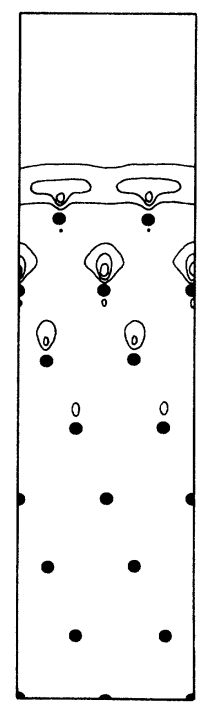

bridge site

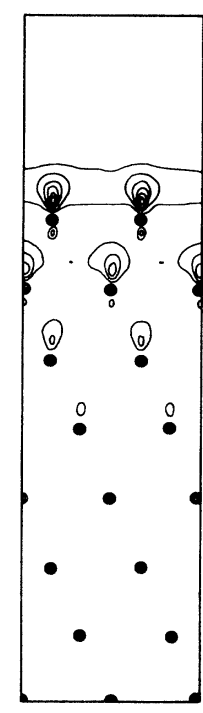

hcp site
FIG. 9. Contour plots of the charge density of the surface state $A$ in the plane formed by the vectors [111] and [11 $\overline{2}]$ with the top layer atoms at (a) the fcc sites, (b) the bridge sites, and (c) the hcp sites. 
tions of the atoms are indicated by filled black circles. From the plot we see that the surface state is localized in the top two or three layers, and is indeed rather similar for the three cases considered. We may conclude that the surface electronic structure is only very weakly dependent on the position of the top-layer Au atoms. We also observe that the charge-density profile for this surface state becomes rather smooth at a short distance above the top layer. Thus, we cannot rationalize the atomic resolution obtained by the STM by the charge-density corrugation of surface states near the Fermi energy. Strong tip and surface interaction or local elastic deformation of the surface by the tip may be other possible explanations that should not be overlooked.

\section{SUMMARY}

We have used first-principles calculations to obtain the energies of the top layer of Au atoms occupying the fcc, hcp, top, and bridge sites on the $\mathrm{Au}(111)$ surfaces. For each of the geometries considered, the atomic positions are fully relaxed with Hellmann-Feynman forces. We found that the fcc and hcp sites are almost degenerate in energy, with the hcp site higher in energy by only $1 \mathrm{mRy}$ per surface atom. The knowledge of the energy of occu- pation of a few high-symmetry sites allow us to extract the surface potential "seen" by the top-layer atoms. Combined with our previous results of a Au monolayer contraction on top of a homogeneous medium (jellium surface), we can use a Frenkel-Kontorowa model to describe the contractive reconstruction on the $\mathrm{Au}(111)$ surface. The equilibrium configuration of this model is found numerically, resulting in a top-layer configuration very similar to the one deduced from experiments. In particular, we found that the top layer contracts along a [110] direction by $5 \%$, and both fcc and hcp sites are occupied in the final configuration. The surface electronic structure of the Au(111) surface is also investigated, and we found that the surface band structure is rather insensitive to the position of the top layer.

\section{ACKNOWLEDGMENTS}

Ames Laboratory is operated for the U.S. Department of Energy by Iowa State University under Contract No. W-7405-ENG-82. This work is supported by the Director of Energy Research, Office of Basic Energy Sciences. U.S. Department of Energy (including a grant of computer time on the Cray computers at the Lawrence Livermore Laboratory).
${ }^{1}$ M. A. Van Hove, R. J. Koestner, P. C. Stair, J. P. Biberian, L. L. Kesmodel, I. Bartos, and G. A. Somorjai, Surf. Sci. 103, 189 (1981).

${ }^{2}$ J. Perdereau, J. P. Biberian, and G. E. Rhead, J. Phys. F 4, 798 (1974).

${ }^{3}$ H. Melle and E. Menzel, Z. Naturforsch. Teil A 33, 282 (1978).

${ }^{4} J$. C. Heyraud and J. J. Metois, Surf. Sci. 100, 519 (1980).

${ }^{5} \mathrm{~K}$. Takayanagi and K. Yagi, Trans. Jpn. Inst. Met. 24, 337 (1983).

${ }^{6}$ U. Harten, A. M. Lahee, J. Peter Toennies, and Ch. Woll, Phys. Rev. Lett. 54, 2619 (1985).

${ }^{7}$ M. El-Batanouny, S. Burdick, K. M. Martini, and P. Stancioff, Phys. Rev. Lett. 58, 2762 (1987).

${ }^{8}$ Ch. Wöll, S. Chiang, R. J. Wilson, and P. H. Lippel, Phys. Rev. B 39, 7988 (1989).

${ }^{9}$ N. Takeuchi, C. T. Chan, and K. M. Ho, Phys. Rev. Lett. 63, 1273 (1989).

10J. Frenkel and T. Kontorowa, Phys. Z. Sowjetunion 13, 1 (1938); F. C. Frank and J. H. van der Merwe, Proc. Soc. London Ser. A 198, 205 (1949).
${ }^{11}$ D. R. Hamann, M. Schlüter, and C. Chiang, Phys. Rev. Lett. 43, 1494 (1979); G. B. Bachelet and M. Schlüter, Phys. Rev. B 25, 2103 (1982); L. Kleinman, ibid. 21, 2630 (1980).

${ }^{12}$ P. Hohenberg and W. Kohn, Phys. Rev. 136, B864 (1964); W. Kohn and L. J. Sham, ibid. 140, A1133 (1965).

${ }^{13}$ L. Hedin and B. I. Lundqvist, J. Phys. C 4, 2064 (1971).

${ }^{14}$ S. G. Louie, K. M. Ho, and M. L. Cohen, Phys. Rev. B 19, 1774 (1979).

${ }^{15}$ C. H. Xu, K. M. Ho, and K. P. Bohnen, Phys. Rev. B 39, 5599 (1989).

${ }^{16}$ N. Takeuchi, C. T. Chan, and K. M. Ho, Phys. Rev. B 40, 1565 (1989).

${ }^{17}$ K. M. Ho, and K. P. Bohnen, Phys. Rev. Lett. 59, 1833 (1987).

${ }^{18}$ D. D. Chambliss and R. J. Wilson (unpublished).

${ }^{19}$ K. G. Huang, D. Gibbs, D. M. Zehner, A. R. Sandy, and S. G. J. Mochrie (unpublished).

${ }^{20}$ The points $K_{1}$ and $K_{2}$ are the same. Only the directions $\Gamma-K 1$ and $\Gamma-K_{2}$, and $M_{1}-K_{1}$ and $M_{2}-K_{2}$ are different.

${ }^{21}$ S. D. Kevan, and R. H. Gaylord, Phys. Rev. B 36, 5809 (1987). 